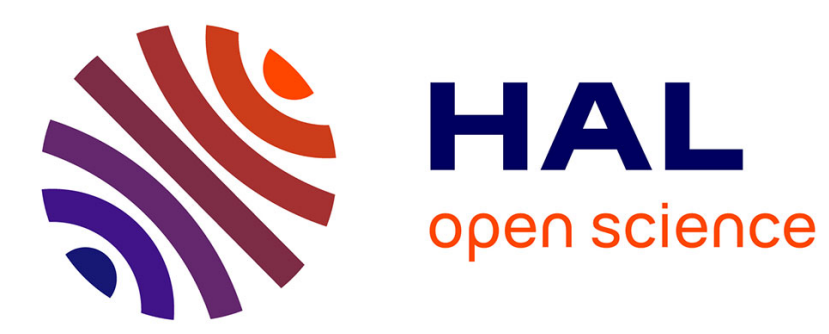

\title{
Reducing the background anisotropy by using hexagonal Fourier transform in two-dimensional turbulent flows
}

J. Chai, Le Fang, Jean-Pierre Bertoglio

\section{To cite this version:}

J. Chai, Le Fang, Jean-Pierre Bertoglio. Reducing the background anisotropy by using hexagonal Fourier transform in two-dimensional turbulent flows. Computers and Fluids, 2020, 210, pp.104671. 10.1016/j.compfluid.2020.104671 . hal-03234692

\section{HAL Id: hal-03234692 \\ https://hal.science/hal-03234692}

Submitted on 9 Jun 2021

HAL is a multi-disciplinary open access archive for the deposit and dissemination of scientific research documents, whether they are published or not. The documents may come from teaching and research institutions in France or abroad, or from public or private research centers.
L'archive ouverte pluridisciplinaire HAL, est destinée au dépôt et à la diffusion de documents scientifiques de niveau recherche, publiés ou non, émanant des établissements d'enseignement et de recherche français ou étrangers, des laboratoires publics ou privés. 


\title{
Reducing the background anisotropy by using hexagonal Fourier transform in two-dimensional turbulent flows
}

\author{
J. Chai, ${ }^{1}$ L. Fang,,${ }^{1, *}$ and J.-P. Bertoglio ${ }^{2}$ \\ ${ }^{1}$ LMP, Ecole Centrale de Pékin, Beihang University, Beijing 100191, China \\ ${ }^{2}$ LMFA, CNRS, Ecole Centrale de Lyon-Université de Lyon, 69130 Ecully, France
}

(Dated: February 4, 2020)

\begin{abstract}
The two-dimensional hexagonal Fourier transform is introduced to reduce the background anisotropy in the numerical calculations with periodic conditions. Comparing to traditional Fourier transform with cartesian mesh, the largescale background anisotropy is significantly reduced under different energy spectra. In addition, the phenomenon of small-scale background anisotropy is found to be related to the type of low-pass filter. Considering the standard deviation of secondorder velocity correlation function with fixed two-point distance, the scaling at small scales is a result of Taylor expansion, while the scaling at large scales can be explained by using a simplified single-wavenumber analytical model. The hexagonal Fourier transform can be easily implemented by using fast Fourier transform (FFT) libraries, and is expected to be applied in future pseudo-spectral methods of two-dimensional and quasi-three-dimensional turbulent flows to minimize the background anisotropy.
\end{abstract}

Keywords: Homogeneous isotropic turbulence; two-dimensional turbulence; large-scale anisotropy; pseudo-spectral methods; direct numerical simulation

\footnotetext{
*le.fang@zoho.com
} 


\section{INTRODUCTION}

The isotropy conditional has been widely used in many fundamental studies of turbulence as a basic assumption. In order to numerically generate isotropic turbulence, the most common method is to present the continuous physical field via a number of discrete points and to involve periodic conditions[1]. However, periodic conditions will also yield a-posteriori largescale effects and reduce the reliablity of direct numerical simulation (DNS) databases[2]. In a previous study, we attributed the large-scale effects to the a-priori background scalar-level anisotropy, which is mainly related to the shape of energy spectrum[3]. We pointed out that enlarging the wavenumber of the energy-containing peak can significantly reduce the background anisotropy. In practice, this means that the energy-containing scale $\mathcal{L}$ should be much smaller than the period of computational domain $R$. For example, in Ref. [2] it was suggested to use $\mathcal{L}<0.1 R$ to obtain negligible large-scale effect. However, in practice this criterion is difficult to be satisfied because of the limitation of computation resources, for example in literature it is usually $\mathcal{L} \sim 0.25 R[4-7]$ which does not satisfy the suggestion of Ref. [2]. Therefore, it would be substantial to investigate a method to reduce the background scalar-level anisotropy without changing the location of the energy-containing peak. In the following, we will briefly revisit our previous understanding on the background scalar-level anisotropy, which directly leads to the idea of the present contribution.

In a turbulence field, the concept of anisotropy can be classified to two levels, i.e., the scalar level and the tensor level. In Ref. [3] we remarked that the scalar-level anisotropy is the precondition to discuss the tensor-level anisotropy. In the present contribution we will focus on the scalar-level anisotropy, since it is a background phenomenon directly affected by the periodic condition. Specifically, in statistically homogeneous turbulence, we can select the second-order structure function, defined as

$$
R_{\theta \theta}(\vec{r}):=\langle\theta(\overrightarrow{0}) \theta(\vec{r})\rangle
$$

where $\theta$ is a scalar, \langle\rangle is ensemble average and $\vec{r}$ is the two-point distance. The scalar-level isotropy then implies that the value of $R_{\theta \theta}(\vec{r})$ should be independent to the direction of $\vec{r}$, i.e., $R_{\theta \theta}(\vec{r})=R_{\theta \theta}(r)$ with $r=|\vec{r}|$. However it is indeed mathematically impossible if we assume periodic conditions (see Ref. [8] for a proof). In order to quantitatively estimate this background scaler-level anisotropy, we simply introduced the standard deviation over a 
spherical surface with radius $r$, written as

$$
\mathcal{A}(r)=\frac{1}{R_{i i}(\overrightarrow{0})} \sqrt{\frac{2}{\pi} \int_{\theta=0}^{\pi / 2} \int_{\phi=0}^{\pi / 2}\left[R_{i i}(r, \theta, \phi)-\mu(r)\right]^{2} \sin \theta \mathrm{d} \phi \mathrm{d} \theta}
$$

with $\mu(r)$ the surface average of the scalar field under spherical coordinate $R_{i i}(r, \theta, \phi)$ :

$$
\mu(r)=\frac{2}{\pi} \int_{\theta=0}^{\pi / 2} \int_{\phi=0}^{\pi / 2} R_{i i}(r, \theta, \phi) \sin \theta \mathrm{d} \phi \mathrm{d} \theta
$$

By using these definitions, we can observe that in a periodic box, the axis direction and diagonal directions show obvious differences, indicating a background anisotropy (see Fig. 1 of Ref. [3] for example). This background anisotropy is indeed because the calculation domain is a square or a cube, rather than ideally a circle or a sphere which has no direction difference. Undoubtedly, using Fourier expansion always implies periodicity and cannot be applied in a circular or spherical domain. However in the present contribution, we are aiming at searching for an alternative method to reduce the background scalar-level anisotropy, instead of eliminating it.

The periodicity is introduced by the discrete low-wavenumber truncation in Fourier spectral space, therefore a natural idea is to reselect the discrete wavevectors which are more isotropic. We remark that this selection cannot be arbitrary in the simulation of turbulent flows. In fact, because of the nonlinear term of Navier-Stokes (NS) equation, any two wavevectors $\vec{p}$ and $\vec{q}$ can exchange energy with the wavevector $\vec{p}+\vec{q}$ via triad interactions[9]. Hence, if $\mathcal{K}$ is the set of discrete wavevectors, $\mathcal{K}$ must be a discrete group. In traditional discrete Fourier analysis (DFT), all wavevectors are selected at cartesian mesh points, which naturally form a group. We notice that in two-dimensional space, regular triangles can also fill the plane, which means that the vertices of regular hexagons can form another discrete group. We remark here that regular tetrahedrons cannot fill a three-dimensional space. Although polyhedra can fill[10, 11], the vertices at polyhedra do not form a group. Therefore, in the present contribution we will show that if we select the discrete wavevectors at this vertices, a hexagonal periodicity will be implied instead of square periodicity. In Sec. II we will introduce the method of hexagonal Fourier transform with these discrete wavenumbers. In Sec. III we will show that the present method can effectively reduce the background scalar-level anisotropy by comparing to traditional cartesian mesh. 


\section{THEORETICAL ANALYSIS}

\section{A. Hexagonal Fourier transform}

A few researchers have used the idea of hexagonal Fourier transform in the domain of signal processing. Murphy and Gallagher [12] studied the hexagonal sampling techniques including the fundamental period of sampling. They selected the sample points via hexagonal mesh in physical space and transformed it to cartesian mesh in spectral space via a modified fast Fourier transform (FFT). Ehrhardt [13] introduced another fast algorithm to transform the physical-space hexagonal samplings to rectangular spectrum output without interpolation. Other existing studies, such as Refs. [14-16], focus on constructing low-level fast algorithms. Our problem is opposite by comparing to these existing studies, since we start from discrete hexagonal wavevectors in spectral space due to the purpose of reducing background anisotropy. In the following parts we will define our concept of hexagonal Fourier transform and introduce a pertinent fast algorithm.

For a two-dimensional scalar field $\theta(\vec{r})$ which is quadratically integrable, the general formulas of the forward and inverse continuous Fourier transforms are respectively

$$
\begin{aligned}
& \hat{\theta}(\vec{k})=\mathscr{F}_{\mathrm{c}}(\theta(\vec{r}))=\frac{1}{4 \pi^{2}} \int_{\vec{r}} \theta(\vec{r}) e^{-i \vec{k} \cdot \vec{r}} d \vec{r}, \\
& \theta(\vec{r})=\mathscr{F}_{\mathrm{c}}^{-1}(\hat{\theta}(\vec{k}))=\int_{\vec{k}} \hat{\theta}(\vec{k}) e^{i \vec{k} \cdot \vec{r}} d \vec{k},
\end{aligned}
$$

where $\hat{\theta}(\vec{k})$ is a scalar at wavevector $\vec{k}$ in Fourier spectral space. If the scalar field can be expressed as a summation of sinusoidal and cosinusoidal functions at discrete wavelengths, a discrete version of Fourier transform can be used. For example, if the set of discrete wavevectors is denoted as $\mathcal{K}$, the inverse Fourier transform can reconstruct the physicalspace field via the form of Fourier series

$$
\theta(\vec{r})=\mathscr{F}^{-1}(\hat{\theta}(\vec{k}))=\sum_{\vec{k} \in \mathcal{K}} \hat{\theta}(\vec{k}) e^{i \vec{k} \cdot \vec{r}}
$$

Inspired from Ref. [12], here we select the discrete wavevectors at the vertices of regular triangles in spectral space. We will start from the case with infinity number of wavevectors without high-wavenumber truncation. As mentioned in Sec. I, this set of discrete wavevectors form a group, which will not generate any harmonic wave outside this group under 
non-linear operations. In the following we will illustrate that the corresponding physical space is periodic in three directions respectively.

A sketch of the discrete wavevectors in spectral space is shown in Fig. 1. Denoting the minimal distance in $k_{1}$-axis as $\Delta_{k}$, the positions of these discrete wavevectors generally write

$$
\mathcal{K}=\left\{\vec{k} \mid \vec{k}=n_{1}\left(\Delta_{k}, 0\right)+n_{2}\left(\frac{\Delta_{k}}{2}, \frac{\sqrt{3} \Delta_{k}}{2}\right), n_{1}, n_{2} \in \mathbb{Z}\right\},
$$

and the inverse DFT (6) becomes

$$
\theta(\vec{r})=\sum_{\vec{k} \in \mathcal{K}} \hat{\theta}(\vec{k}) \exp (i \mathcal{G}(\vec{r}, \vec{k}))
$$

with

$$
\mathcal{G}(\vec{r}, \vec{k})=r_{1} n_{1} \Delta_{k}+r_{1} n_{2} \frac{\Delta_{k}}{2}+r_{2} n_{2} \frac{\sqrt{3} \Delta_{k}}{2}
$$

It is easy to verify that there are three periodicity vectors for $\mathcal{G}(\vec{r}, \vec{k})$ in physical space, s.t. $\forall n_{1}, n_{2} \in \mathbb{Z}, \vec{k} \in \mathcal{K}, \mathcal{G}(\vec{R}, \vec{k}) \equiv \mathcal{G}(\overrightarrow{0}, \vec{k})$. From Eq. (8) this will directly leads to $\theta(\vec{R})=\theta(\overrightarrow{0})$. These periodicity vectors are respectively

$$
\begin{aligned}
& \vec{R}_{1}=\left(0, \frac{4 \pi}{\sqrt{3} \Delta_{k}}\right), \\
& \vec{R}_{2}=\left(\frac{2 \pi}{\Delta_{k}}, \frac{2 \pi}{\sqrt{3} \Delta_{k}}\right), \\
& \vec{R}_{3}=\left(\frac{2 \pi}{\Delta_{k}},-\frac{2 \pi}{\sqrt{3} \Delta_{k}}\right),
\end{aligned}
$$

which are all of length $\frac{4 \pi}{\sqrt{3} \Delta_{k}}$. Note that the traditional DFT at cartesian mesh yields a period $\frac{2 \pi}{\Delta_{k}}$ with $\Delta_{k}$ the minimal distance in spectral space, which is shorter than the present hexagonal case. As discussed in Ref. [3], the period is the only characteristic scale in physical space and is used in normalization, thus larger period will generally reduce the anisotropy for the same scale in physical space. In addition, here we have three periods, which are more than the two periods in traditional DFT at cartesian mesh. As discussed in Sec. I, this implies that the present case is more close to the ideal spherical computational domain, thus it should be intuitively less anisotropic than traditional cartesian mesh.

We also developed a fast numerical algorithm for the numerical calculation of the present hexagonal Fourier transform. In order to avoid scattering the main topic of the present contribution, the detailed numerical algorithm is shown in Appendix A. Our algorithm has 


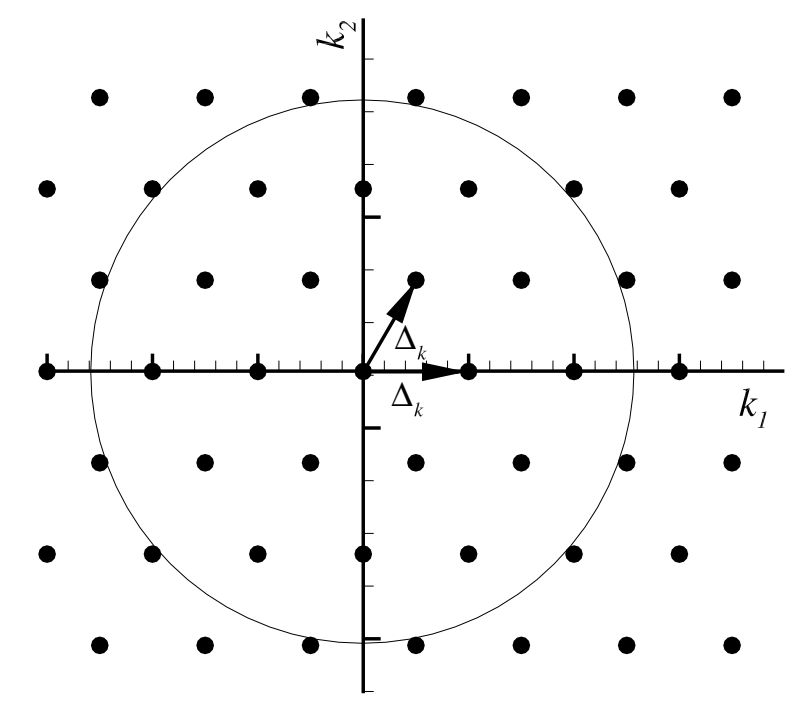

Figure 1. Locations of the discrete wavevectors in two-dimensional spectral space. $N / 2=3$ is selected as an example. The circle indicates the isotropic low-pass filter introduced in Eq. (16).

the same algorithm complexity as existing studies[14-16], and it is easier to be implemented in real calculations by employing existing parallelizable FFT libraries.

In the following parts we will then quantitatively estimate the background scalar-level anisotropy in two-dimensional turbulence.

\section{B. Correlation function in homogeneous turbulence}

In previous parts, the background scalar-level anisotropy is explained by involving a scalar field $\theta$. In homogeneous turbulence, this should correspond to a coordinate invariant of velocity-velocity tensors. A most simple scalar field can be the second-order summation correlation function $R_{i i}(\vec{r}):=\left\langle u_{i}(\overrightarrow{0}) u_{i}(\vec{r})\right\rangle$, with $\vec{u}$ velocity vector. The discrete wavevectors in spectral space are selected at a hexagonal mesh described in previous subsections with $\Delta_{k}=2 \pi / L$ with $L$ a characteristic length. Considering practical calculations with finite mesh resolution, we introduce high-wave-number numerical truncation, which means that in both $k_{1}$ and $k_{2}$ directions there are $N+1$ discrete wavevectors, centered at 0 . Similar to 
Appendix A, the set of these wavevectors writes

$$
\begin{gathered}
\mathcal{K}=\left\{\frac{2 \pi}{L}(2 p, 2 q), \frac{2 \pi}{L}(2 p+1,2 q), \frac{2 \pi}{L}\left(2 p+\frac{1}{2}, 2 q+1\right), \frac{2 \pi}{L}\left(2 p+\frac{3}{2}, 2 q+1\right) \mid\right. \\
\left.p=-\frac{N}{2},-\frac{N}{2}+1, \ldots,-1,0,1, \ldots, \frac{N}{2}-1, \frac{N}{2}, q=\frac{\sqrt{3}}{2} p\right\} .
\end{gathered}
$$

Figure 1 is a sketch for these wavevectors. The velocity in spectral space is denoted as $\overrightarrow{\hat{u}}$, and the energy spectrum over these discrete points is defined as an energy spectrum $E(k):=\sum_{\vec{z} \in \mathcal{K}, z=k} \frac{1}{2} \hat{u}_{i}(\vec{z}) \hat{u}_{i}^{*}(\vec{z})$ with $\bullet *$ conjugation and $k=|\vec{k}|$. Note that the energy spectrum defined at discrete wavevectors is slightly different with the continuous spectrum, see Ref. [17] for a detailed discussion.

By using the properties of Fourier transform and the homogeneous condition[18], we can write the relations for the correlation function $R_{i i}$ in two dimensional plane

$$
\begin{aligned}
R_{i i}(\vec{r}) & =\left\langle u_{i}(\overrightarrow{0}) u_{i}(\vec{r})\right\rangle=\sum_{\vec{k} \in \mathcal{K}}\left\langle\hat{u}_{i}(\vec{k}) \hat{u}_{i}^{*}(\vec{k})\right\rangle e^{i \vec{k} \cdot \vec{r}} \\
& =\sum_{\vec{k} \in \mathcal{K}} \frac{E(k)}{\pi k} e^{i \vec{k} \cdot \vec{r}}
\end{aligned}
$$

where

$$
\hat{u}_{i}(\vec{k})=\mathscr{F}\left(u_{i}(\vec{r})\right)=\frac{1}{4 \pi^{2}} \int_{\vec{r}} u_{i}(\vec{r}) e^{-i \vec{k} \cdot \vec{r}} d \vec{r} .
$$

We also remark that besides the summation correlation functions, there can be other coordinate invariants which can be used as a scalar field. However, it is usually difficult to relate them to some commonly-known quantities. For instance, Gaussian assumption implies that the fourth-order correlation functions can be represented by second-order correlation functions, but in real turbulence the accumulation terms are also not negligible[19-21]. In addition, these high-order invariants are even usually less anisotropic than low-order invariants. Hence, the second-order summation correlation function considered in the present contribution can be considered as a minimum request for background scalar-level isotropy.

Similar to Eq. (2), in two-dimensional turbulence we can define the standard deviation over a circle to quantitatively estimate the background scalar-level anisotropy, that is

$$
\mathcal{A}(r)=\frac{1}{R_{i i}(\overrightarrow{0})} \sqrt{\frac{1}{2 \pi} \int_{\theta=0}^{2 \pi}\left[R_{i i}(r, \theta)-\mu(r)\right]^{2} \mathrm{~d} \theta},
$$

with $\mu(r)$ the surface average of the scalar field under spherical coordinate $R_{i i}(r, \theta)$

$$
\mu(r)=\frac{1}{2 \pi} \int_{\theta=0}^{2 \pi} R_{i i}(r, \theta) \mathrm{d} \theta .
$$




\section{RESULTS}

In this section, we will show that using the hexagonal Fourier transform in spectral discretization can effectively reduce the background scalar-level anisotropy. In Sec. III A we will give a quantitative overview, focusing on the influences of resolution and energy spectrum. Comparison with traditional cartesian mesh will be presented in Sec. IIIB to emphasize the advantage of the present method. In Sec. IIIC we will introduce a simple analytical model to provide a quantitative explanation on the contribution of single wavenumber to background anisotropy.

\section{A. Background anisotropy and influence of energy spectrum under hexagonal Fourier transform}

We perform numerical cases to quantitatively show this background scalar-level anisotropy. We select the maximum wavenumber $N / 2=64$ (corresponding to a $128^{2}$ resolution) by employing an isotropic low-pass filter (see Fig. 1 as a sketch) and apply the traditional Kolmogorov $-5 / 3$ energy spectrum at the discrete wavenumbers, that is

$$
E(k)= \begin{cases}0, & \text { if } k=0 \text { or } k>\sqrt{3} \pi N /(2 L), \\ k^{-5 / 3}, & \text { otherwise. }\end{cases}
$$

Figure 2 shows the values of $R_{i i}(\vec{r})$ in the two-dimensional quarter space $\left(r_{1}, r_{2}\right)$ for $r_{1}, r_{2}>0$. The points that $R_{i i}(\vec{r})=1$ correspond to the period of the physical-space field. Two minimum periods $\vec{R}_{1}$ and $\vec{R}_{2}$ in Eq. (10) are shown in the figure. The third period $\vec{R}_{3}$ is in fact a linear combination of $\vec{R}_{1}$ and $\vec{R}_{2}$ since $\vec{R}_{3}=\vec{R}_{1}-\vec{R}_{2}$. Anisotropy can be illustrated in this figure, as in different directions of $\vec{r}$ the values of $R_{i i}(\vec{r})$ change differently. For example, in $r_{1}$-direction the period is $2 L$, while in $r_{2}$-direction it is $2 L / \sqrt{3}$. At small scales it is quasi isotropic, as the isolines are nearly circles. Specifically, we select six typical directions of $\vec{r}$ and show the values of $R_{i i}(\vec{r})$ in Fig. 3 respectively. Note that several curves completely overlap with each other because of hexagonal symmetry. For comparison, in the ideal case where the background anisotropy is not present, all the curves are expected to completely overlap.

As explained in Ref. [3], the resolution of discretization scarcely contributes to the large-scale background anisotropy. The reason is that different resolutions lead to small- 


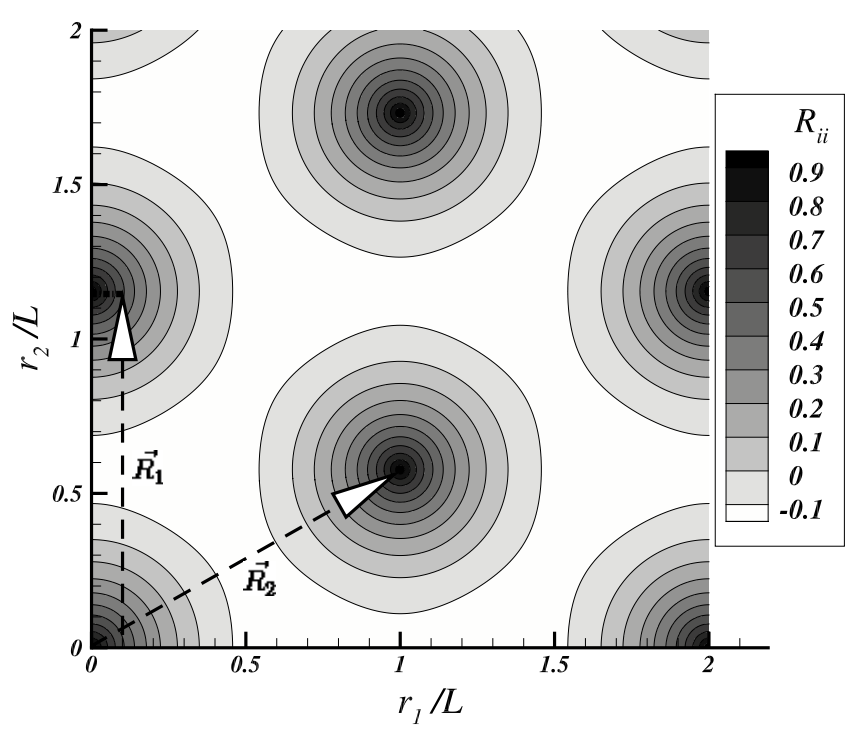

Figure 2. Values of $R_{i i}(\vec{r})$ in quarter space $\left(r_{1}, r_{2}\right)$ for $r_{1}, r_{2}>0$. Energy spectrum is assumed to be Eq. (16). The half-grid number is $N / 2=64 . R_{i i}(\overrightarrow{0})$ is used for normalization.

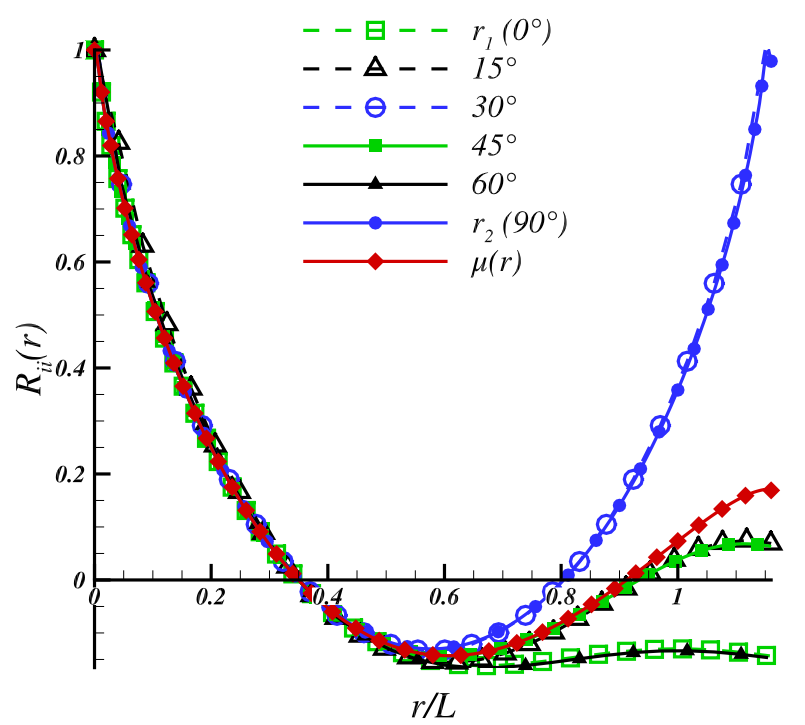

Figure 3. $R_{i i}(\vec{r})$ along $r_{1}\left(0^{\circ}\right), 15^{\circ}, 30^{\circ}, 45^{\circ}, 60^{\circ}$ and $r_{2}\left(90^{\circ}\right)$ directions, respectively. The circularaveraged value $\mu(r)$ is also plotted. Energy spectrum is assumed to be Eq. (16). The half-grid number is $N / 2=64 . R_{i i}(\overrightarrow{0})$ is used for normalization. Because of hexagonal symmetry, the results of $30^{\circ}$ and $r_{2}\left(90^{\circ}\right)$ directions are the same, while the results of $r_{1}\left(0^{\circ}\right)$ and $60^{\circ}$ directions are the same. 


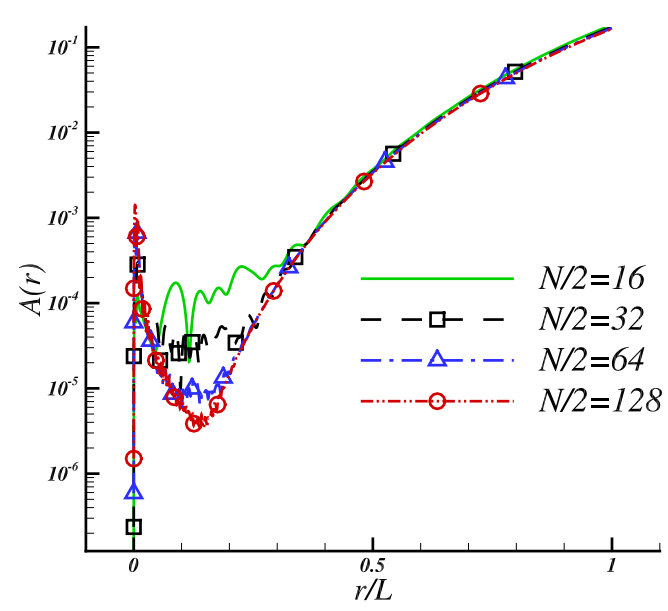

(a)

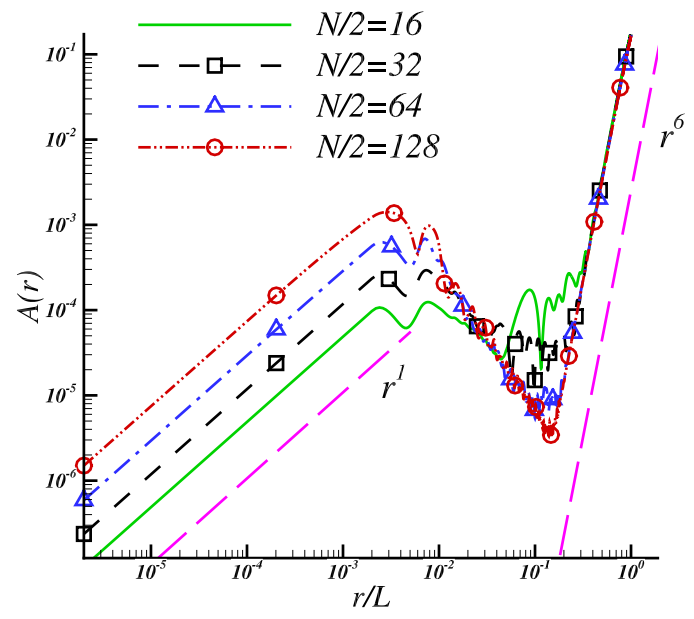

(b)

Figure 4. Standard deviation $\mathcal{A}(r)$ under different half-grid numbers $N / 2=16,32,64$ and 128 respectively. The energy spectrum is Eq. (16). $R_{i i}(\overrightarrow{0})$ is used for normalization. (a) Log-Linear plot. (b) Log-Log plot.

scale differences, but the background anisotropy is a large-scale phenomenon in the order of domain size. In Fig. 4 we compare the results of different resolutions and find that the background anisotropy is almost the same at large scales. Differences exist at small scales, which to our knowledge has not been discussed in literature. In the following part we will show that it is a phenomenon related to high-wavenumber truncation, or say the low-pass filter. In Fig. 4(a) it is observed that higher resolution generally reduces smallscale anisotropy. Figure 4(b) clearly shows that the small- and large-scale anisotropy follows different scalings, indicating the different underlying mechanisms. At very small scales where $r \ll 2 \pi / k_{\max }$, for all resolutions there is $\mathcal{A}(r) \propto r^{1}$ scaling which corresponds to the result of Taylor expansion of standard deviation. This can be explained since it is smaller than the mesh resolution in physical space, which has not been discussed in Ref. [3]. By contrast, at large scales there is another scaling of $\mathcal{A}(r) \propto r^{6}$. We will show in Sec. IIIC that this scaling is closely related to the type of spectral discretization of single wavenumber. The region between these two scalings (approximately at $r / L \sim 0.1$ where we have slight fluctuations and different scalings of $\mathcal{A}(r)$ under different half-grid numbers) should be due to the discretization near the edge of low-pass filter. For illustration, we compare the standard deviation of the present case with the non-filter one in Fig. 5. Clearly, although an isotropic 


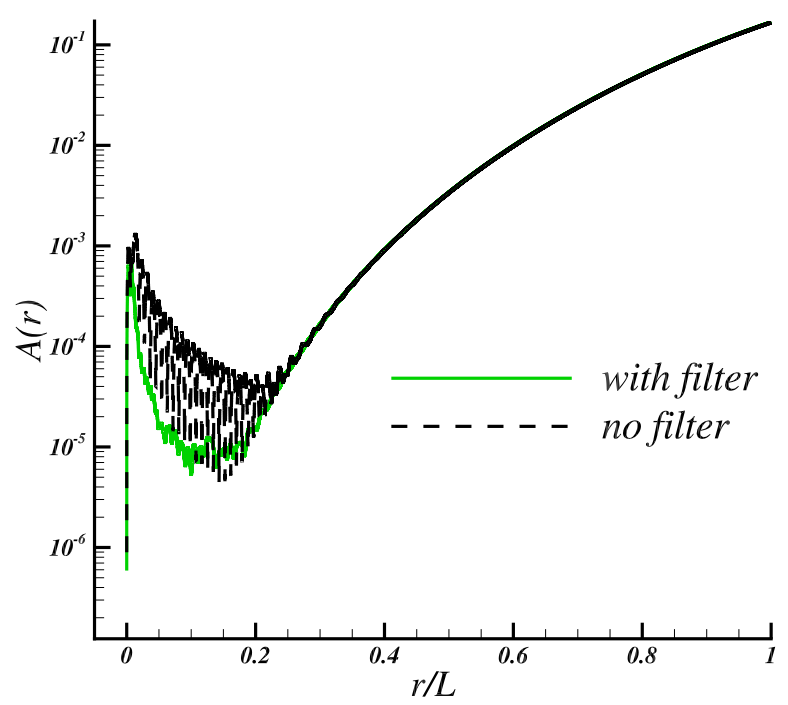

Figure 5. Standard deviation $\mathcal{A}(r)$ with or without filter in spectral space. $R_{i i}(\overrightarrow{0})$ is used for normalization. The energy spectrum is Eq. (16). The half-grid number is $N / 2=64$.

low-pass filter reduces the number of effective wavevectors, the oscillation and the value of $\mathcal{A}(r)$ is significantly reduced in the region that $r / L \sim 0.1$. This result supports the use of low-pass filter in practical applications. In the following parts we therefore will also use the low-pass filter for all cases. In brief, we can say that both low-wavenumber and highwavenumber truncations affect the background anisotropy: the former one is dominant at large scales while the latter one is dominant at small scales. The transition between them is related to the filter type.

From Ref. [3] we know that the shape of the energy-containing scales should contribute much at large scales, therefore we will introduce the peak location $k_{p}$ which corresponds to the location of maximum energy, and write

$$
E(k)= \begin{cases}0, & \text { if } k>\sqrt{3} \pi N /(2 L), \\ k^{2}, & \text { if } k \leq k_{p}(2 \pi / L) \\ k^{-5 / 3}, & \text { otherwise. }\end{cases}
$$

In particular, $k_{p}=1$ is the case corresponding to Eq. (16). Results of different values of $k_{p}$ are shown in Fig. 6. The standard deviation, shown in Fig. 6(b), estimates quantitatively the anisotropy at different scales. It is found in Fig. 6(b) that in general, larger $k_{p}$ leads to less anisotropy at large scales, which is in agreement with Ref. [3] in tendency. It is 


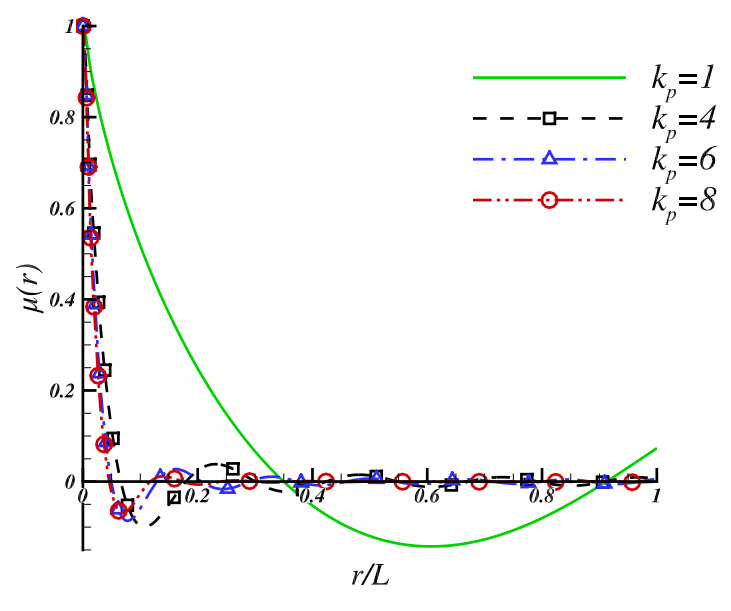

(a)

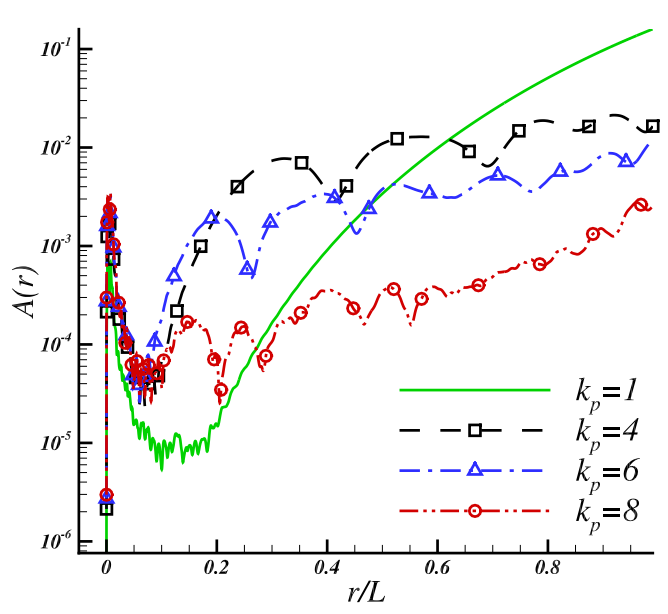

(b)

Figure 6. Comparison of different energy spectra. Energy spectra are Eq. (17) with $k_{p}=1,4,6$ and 8 respectively. $R_{i i}(\overrightarrow{0})$ is used for normalization. The half-grid number is $N / 2=64$. (a) Circular average $\mu(r)$, (b) Standard deviation $\mathcal{A}(r)$.

also found that at small scales, $k_{p}=1$ yields less anisotropy than others. This is also in agreement with Fig. 7(b) of Ref. [3].

To summarize, the background anisotropy by using hexagonal Fourier transform is similar with the case of traditional cartesian mesh in tendency. The shape of energy-containing part is a dominant reason for the background anisotropy, which can be quantitatively presented by using the location of peak $k_{p}$. It is then worth to compare the present results with traditional cartesian mesh in order to illustrate that the present method can reduce the background anisotropy.

\section{B. Comparisons with traditional cartesian mesh}

Following Ref. [3], we calculate similarly the two-dimensional cases by using traditional cartesian mesh in spectral space, and compare them to the present hexagonal Fourier transform. Comparisons are performed under the same number of discrete wavevectors and the same minimal distance $\Delta_{k}$ in spectral space in each case with $N / 2=64$.

For the cases that $k_{p}=1$, the values of $\mathcal{A}(r)$ are shown in Fig. 7(a). Clearly the hexagonal Fourier transform is less anisotropy than the traditional cartesian case at large scales. 


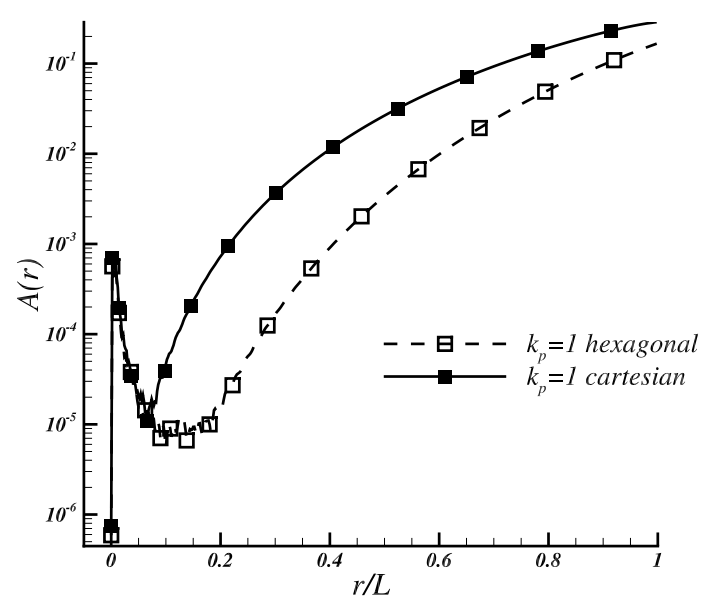

(a)

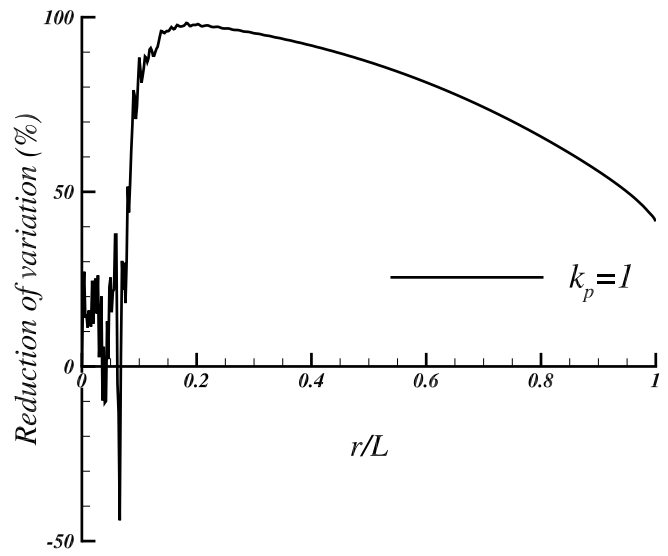

(b)

Figure 7. Comparison on the standard deviation $\mathcal{A}(r)$ for hexagonal and cartesian cases. Energy spectra are Eq. (16) with $k_{p}=1$. The half-grid number is $N / 2=64$. $R_{i i}(\overrightarrow{0})$ is used for normalization. (a) Comparison of $\mathcal{A}(r)$. (b) Reduction of variance, defined as $\left(\mathcal{A}_{\text {cartesian }}(r)-\mathcal{A}_{\text {hexagonal }}(r)\right) / \mathcal{A}_{\text {cartesian }}(r)$.

Quantitative comparison can be performed by defining the relative difference $\left(\mathcal{A}_{\text {cartesian }}(r)-\right.$ $\left.\mathcal{A}_{\text {hexagonal }}(r)\right) / \mathcal{A}_{\text {cartesian }}(r)$. In Fig. $7(\mathrm{~b})$ it is shown that the reduction can be in maximum more than $90 \%$ at medium scales. For large scales there is also more than $40 \%$ reduction. Similar result can be found in Fig. 8 for the cases that $k_{p}=4,6$ and 8 respectively. It is also found that the hexagonal Fourier transform is in general less anisotropic than traditional cartesian cases at large scales. At $r \sim L$ the hexagonal Fourier transform leads to more than $70 \%$ reduction of background anisotropy in these cases. There are several points at medium and small scales where hexagonal Fourier transform yields greater anisotropy than cartesian mesh, but in general the reduction of anisotropy is significant, in particular at large scales.

\section{A simplified analytical model}

It is very difficult to derive general formulas for the background anisotropy under hexagonal Fourier transform. Instead, we introduce a simple analytical model to quantitatively show the effect of individual wavenumbers. Considering the case with only six wavenumbers at the vertices of a hexagon. The module of these wavenumbers is denoted as $m k$ where $m$ 


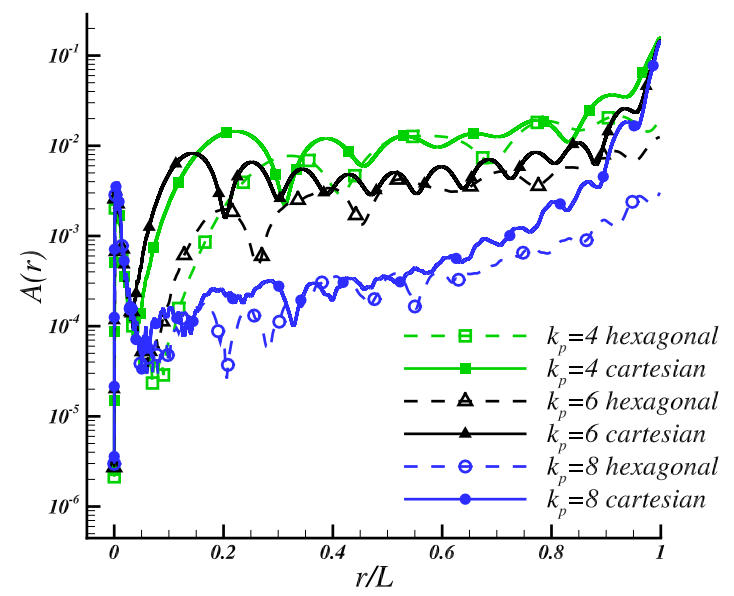

(a)

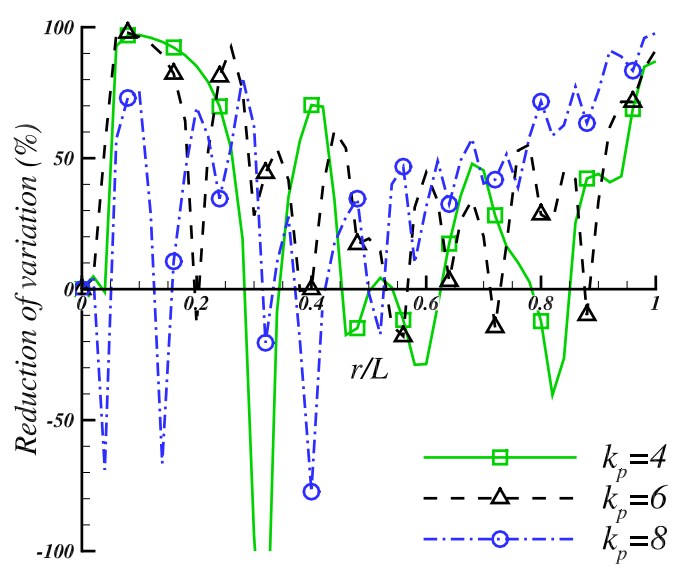

(b)

Figure 8. Comparison on the standard deviation $\mathcal{A}(r)$ for hexagonal and cartesian cases. Energy spectra are Eq. (16) with $k_{p}=4,6$ and 8 respectively. The half-grid number is $N / 2=64$. $R_{i i}(\overrightarrow{0})$ is used for normalization. (a) Comparison of $\mathcal{A}(r)$. (b) Reduction of variance, defined as $\left(\mathcal{A}_{\text {cartesian }}(r)-\mathcal{A}_{\text {hexagonal }}(r)\right) / \mathcal{A}_{\text {cartesian }}(r)$.

is an integer multiplier. The scalar field then write

$$
\theta\left(r_{1}, r_{2}\right)=\cos \left(\frac{\pi}{L} m r_{1}+\frac{\pi \sqrt{3}}{L} m r_{2}\right)+\cos \left(\frac{2 \pi}{L} m r_{1}\right)+\cos \left(\frac{\pi}{L} m r_{1}-\frac{\pi \sqrt{3}}{L} m r_{2}\right)
$$

Due to the condition of spatial homogeneity, it is possible to translate the ensemble average operator to spatial average (similar ideas can be found in Refs. [3, 22]). We choose an area which can represent one period of the scalar field and we are going to calculate the correlation function based on the average of this area. The shape of this area is the hexagonal form, denoted as $\mathcal{D}$, with length of a side $2 L / \sqrt{3}$, and one of its axis coinciding with axis $r_{2}$.

Three typical directions, i.e., $r_{1}\left(0^{\circ}\right), 15^{\circ}$ and $30^{\circ}$ directions, are selected to calculate the correlation analytically. As was shown in Fig. 3, other typical directions are the same to one of the three directions due to hexagonal symmetry. The corresponding values of $R_{\theta \theta}$ are 
denoted as $R_{\theta \theta}^{(1)}, R_{\theta \theta}^{(2)}$ and $R_{\theta \theta}^{(3)}$ respectively. We finally obtain

$$
\begin{aligned}
R_{\theta \theta}^{(1)}(r) & =\frac{1}{S_{\mathcal{D}} R_{\theta \theta}(\overrightarrow{0})} \iint_{\mathcal{D}} \theta\left(r_{1}+r, r_{2}\right) \theta\left(r_{1}, r_{2}\right) \mathrm{d} r_{1} \mathrm{~d} r_{2} \\
& =\frac{2}{3} \cos \left(\frac{m \pi r}{L}\right)+\frac{1}{3} \cos \left(\frac{2 m \pi r}{L}\right) \\
R_{\theta \theta}^{(2)}(r) & =\frac{1}{S_{\mathcal{D}} R_{\theta \theta}(\overrightarrow{0})} \iint_{\mathcal{D}} \theta\left(r_{1}+\frac{\sqrt{2}}{2} r, r_{2}+\frac{\sqrt{2}}{2} r\right) \theta\left(r_{1}, r_{2}\right) \mathrm{d} r_{1} \mathrm{~d} r_{2} \\
& =\frac{1}{3}\left[\cos \left(\frac{\sqrt{2} m \pi r}{L}\right)+\cos \left(\frac{(-1+\sqrt{3}) m \pi r}{\sqrt{2} L}\right)+\cos \left(\frac{(1+\sqrt{3}) m \pi r}{\sqrt{2} L}\right)\right], \\
R_{\theta \theta}^{(3)}(r) & =\frac{1}{S_{\mathcal{D}} R_{\theta \theta}(\overrightarrow{0})} \iint_{\mathcal{D}} \theta\left(r_{1}+\frac{\sqrt{3}}{2} r, r_{2}+\frac{1}{2} r\right) \theta\left(r_{1}, r_{2}\right) \mathrm{d} r_{1} \mathrm{~d} r_{2} \\
& =\frac{1}{3}\left[1+2 \cos \left(\frac{\sqrt{3} m \pi r}{L}\right)\right]
\end{aligned}
$$

where

$$
R_{\theta \theta}(\overrightarrow{0})=\frac{1}{S_{\mathcal{D}}} \iint_{\mathcal{D}} \theta\left(r_{1}, r_{2}\right)^{2} \mathrm{~d} r_{1} \mathrm{~d} r_{2}
$$

is employed for normalization and $S_{\mathcal{D}}$ is the surface of the $\mathcal{D}$. We select two different values of $m$ respectively and show the correlation function at these three typical directions in Fig. 9. Clearly small scales overlap and large scales separate, corresponding to the fact of largescale background anisotropy. This indicates that one single wavelength can already lead to background anisotropy. In fact, we will show that this extremely simple model can correctly represent the scaling of large-scale background anisotropy.

To investigate the anisotropy, an extreme simplification is to use $R_{\theta \theta}^{(1)}(r), R_{\theta \theta}^{(2)}(r)$ and $R_{\theta \theta}^{(3)}(r)$ as three samples with fixed $r$ and calculate their standard deviation $\sigma_{\text {hexagonal ana- }}$ lytically. The analytical form of this standard deviation $\sigma_{\text {hexagonal }}(r)$ is complicated, but its Taylor expansion at $r / L$ simply leads to

$$
\sigma_{\text {hexagonal }}=\frac{\pi^{6}}{360}\left(\frac{m r}{L}\right)^{6}+\frac{\pi^{8}}{2520}\left(\frac{m r}{L}\right)^{8}+\frac{\pi^{10}}{40320}\left(\frac{m r}{L}\right)^{10}+O\left(\left(\frac{m r}{L}\right)^{12}\right),
$$

indicating that the dominant order should be $r^{6}$. This standard deviation $\sigma_{\text {hexagonal }}(r)$ is a statistical result over three samples on a circle, but it might be qualitatively analogous to $\mathcal{A}(r)$ which is defined when the number of samples tends to infinity. In fact, in Fig. 10(a) we show that this scaling is in nice agreement with the real spectrum result presented in Sec. III A. Note that here the scaling $r^{6}$ cannot be applied at the very small scales, since it is the subgrid effect which dominate while this model implies extremely fine mesh. This indicates 


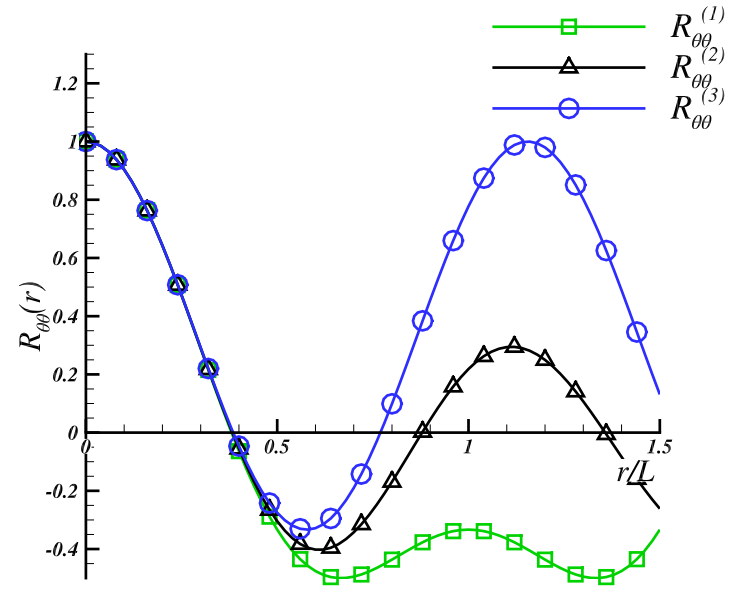

(a)

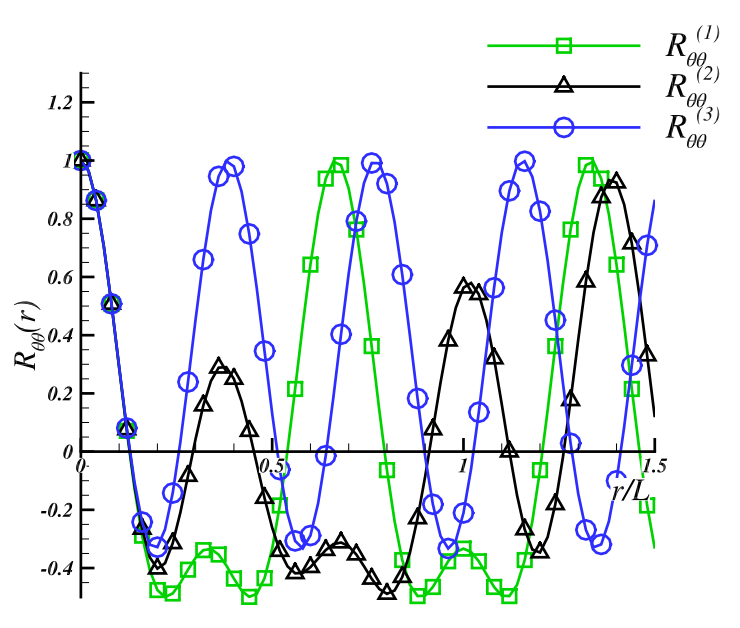

(b)

Figure 9. Illustration of the simple analytical model of correlation function normalized in three typical directions. $R_{\theta \theta}(\overrightarrow{0})$ is used for normalization. a) $m=1$, b) $m=3$.

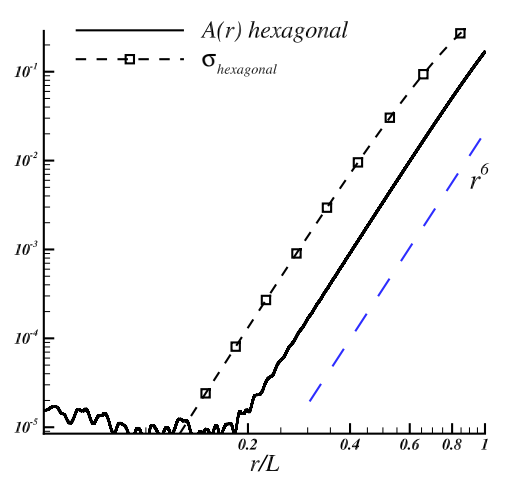

(a)Hexagonal case

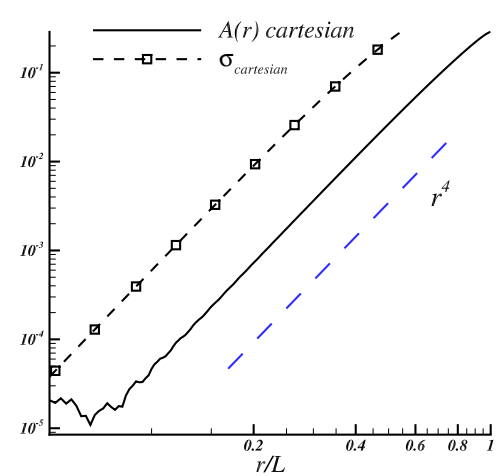

(b)Cartesian case

Figure 10. Comparison of standard deviation $\mathcal{A}(r)$ with real energy spectrum (16) to the threesample standard deviation $\sigma_{\text {hexagonal }}$ and $\sigma_{\text {cartesian }}$ in the single-wavenumber analytical model with $m=1$. $R_{i i}(\overrightarrow{0})$ is used for normalization. The half-grid number is $N / 2=64$. (a) Hexagonal case, (b) Cartesian case.

that the discretization of single wavenumber is the dominant contribution for large-scale background anisotropy.

For comparison, we can also write the case with cartesian discrete points in spectral space. Similarly, three typical directions are also selected as the $r_{1}\left(0^{\circ}\right), 22.5^{\circ}$, and $45^{\circ}$ 
directions. The corresponding correlation functions $R_{\theta_{c} \theta_{c}}(\vec{r})$ for the traditional cartesian case are then respectively

$$
\begin{aligned}
R_{\theta_{c} \theta_{c}}^{(1)}(r) & =\frac{1}{4 L^{2} R_{\theta_{c} \theta_{c}}(\overrightarrow{0})} \int_{-L}^{L} \int_{-L}^{L} \theta_{c}\left(r_{1}+r, r_{2}\right) \theta_{c}\left(r_{1}, r_{2}\right) \mathrm{d} r_{1} \mathrm{~d} r_{2} \\
& =\frac{1}{2}+\frac{1}{2} \cos \left(\frac{2 m \pi r}{L}\right) \\
R_{\theta_{c} \theta_{c}}^{(2)}(r) & =\frac{1}{4 L^{2} R_{\theta_{c} \theta_{c}}(\overrightarrow{0})} \int_{-L}^{L} \int_{-L}^{L} \theta_{c}\left(r_{1}+\frac{\sqrt{2+\sqrt{2}}}{2} r, r_{2}+\frac{\sqrt{2-\sqrt{2}}}{2} r\right) \theta_{c}\left(r_{1}, r_{2}\right) \mathrm{d} r_{1} \mathrm{~d} r_{2} \\
& =\frac{1}{2} \cos \left(\frac{\sqrt{2+\sqrt{2}} m \pi r}{L}\right)+\frac{1}{2} \cos \left(\frac{\sqrt{2-\sqrt{2}} m \pi r}{L}\right), \\
R_{\theta_{c} \theta_{c}}^{(3)}(r) & =\frac{1}{4 L^{2} R_{\theta_{c} \theta_{c}}(\overrightarrow{0})} \int_{-L}^{L} \int_{-L}^{L} \theta_{c}\left(r_{1}+\frac{\sqrt{2}}{2} r, r_{2}+\frac{\sqrt{2}}{2} r\right) \theta_{c}\left(r_{1}, r_{2}\right) \mathrm{d} r_{1} \mathrm{~d} r_{2} \\
& =\cos \left(\frac{\sqrt{2} m \pi r}{L}\right)
\end{aligned}
$$

where

$$
R_{\theta_{c} \theta_{c}}(\overrightarrow{0})=\frac{1}{4 L^{2}} \iint_{\mathcal{D}} \theta_{c}\left(r_{1}, r_{2}\right)^{2} \mathrm{~d} r_{1} \mathrm{~d} r_{2}
$$

and $\theta_{c}\left(r_{1}, r_{2}\right)$ is the scalar field corresponding to the cartesian case where the spectrum is assumed to be discretized only on two axis directions

$$
\theta_{c}\left(r_{1}, r_{2}\right)=\cos \left(\frac{2 \pi}{L} m r_{1}\right)+\cos \left(\frac{2 \pi}{L} m r_{2}\right)
$$

Taylor expansion of the standard deviation of $R_{\theta_{c} \theta_{c}}^{(1)}(r), R_{\theta_{c} \theta_{c}}^{(2)}(r)$ and $R_{\theta_{c} \theta_{c}}^{(3)}(r)$ is

$$
\sigma_{\text {cartesian }}=\frac{\sqrt{6} \pi^{4}}{36}\left(\frac{m r}{L}\right)^{4}-\frac{\sqrt{6} \pi^{6}}{180}\left(\frac{m r}{L}\right)^{6}+\frac{\sqrt{6} \pi^{8}}{2160}\left(\frac{m r}{L}\right)^{8}+O\left(\left(\frac{m r}{L}\right)^{10}\right)
$$

indicating that the dominant order is $r^{4}$. It is also in nice agreement with real spectrum result in Fig. 10(b), which supports the present simplified analytical model. In addition, by comparing between the hexagonal and cartesian cases, we can conclude that the reduction of background anisotropy by using hexagonal Fourier transform is due to the vanish of low-order terms in the single-wavenumber discretization.

\section{CONCLUDING REMARKS}

In the present study we introduce the hexagonal Fourier transform, which means that the discrete wavenumbers in two-dimensional spectral space are selected in hexagonal forms. 
Forward and inverse transforms are derived to support the calculations. Fast algorithms based on FFT are introduced. According to the relation between correlation function and discrete energy spectrum, the background anisotropy can be related to energy spectrum. We then show that under different energy spectra, the hexagonal Fourier transform yields significantly less anisotropy than traditional cartesian mesh. This result then allows to use greater ratio between integral scale $\mathcal{L}$ and the period of computational domain $R$ in two-dimensional turbulent flows if we use the hexagonal Fourier transform in the pseudospectral simulations. In addition, we discuss the small-scale background anisotropy which was not visited in literature, and show that it is closely related to the type of low-pass filter. Under hexagonal Fourier transform, the scaling of background anisotropy (quantified by the standard deviation $\mathcal{A}(r))$ is $r^{1}$ at small scales and $r^{6}$ at large scales. The former scaling is a result of Taylor expansion at subgrid scales and the latter scaling can be explained by using a simplified single-wavenumber analytical model.

Another perspective of the hexagonal Fourier transform is to provide an alternative spectral decomposition for two-dimensional field. In fact, comparing to traditional cartesian meshes, the hexagonal mesh in spectral space are spiritually more close to the idea of shell models[10, 23-25], and will perhaps inspire new researches on the energy transfer among scales. For instance, a simple approach of the large-eddy simulation might be defined on the hexagonal mesh in spectral space. Specifically, we can select the grid-scale (GS) part with only six wavenumbers, located at the vertices of hexagon with the same wavelength. The other wavenumbers are analogous to subgrid-scale (SGS) part. This would enable energy transfers of both GS-GS and GS-SGS interactions, in which the former is a simplification of the non-linear term of filtered NS equation corresponding to local triad interactions, while the latter corresponds to the subgrid stress tensor. We have already obtained some preliminary analytical models under this hexagon framework. By contrast, traditional cartesian mesh cannot easily produce the GS non-linear interactions.

Finally we remark that the hexagonal Fourier transform is restricted in two dimensions. The reason is that regular triangle can fill a two-dimensional space, but regular tetrahedrons cannot fill a three-dimensional space. However, this does not mean that the hexagonal Fourier transform is restricted in two-dimensional turbulence. In fact, there are other types of turbulent flows which also depends on only two spatial coordinates. As an example, Refs. [26, 27] show a general type of these quasi-three-dimensional flows. From the present 
contribution we can also imagine an artificial method to generate other types of quasitwo-dimensional flows, by keeping all the modes in a spectral plane and clearing all other modes. In brief, for all these two-dimensional or quasi-three-dimensional turbulent flows, the hexagonal Fourier transform is suggested as an efficient tool to reduce large-scale background anisotropy.

\section{ACKNOWLEDGEMENT}

This work is supported by the National Science Foundation in China (Grant No.s 11772032, 11572025, 51420105008).

\section{Appendix A: Fast algorithms of hexagonal Fourier transform}

In this section, we will introduce fast algorithms on the transform between hexagonal discrete points in Fourier space and cartesian discrete points in physical space by using FFT and inverse FFT (IFFT) algorithms.

Let us start from traditional two-dimensional DFT under cartesian meshes. Considering a finite-extent sequence in physical space $x\left(n_{1}, n_{2}\right)$ over a region of support $n_{1}=0 \ldots N_{1}-$ $1, n_{2}=0 \ldots N_{2}-1$, the FFT algorithm calculates its discrete spectrum obtained by DFT in the form of a finite-extent sequence $X^{F}\left(k_{1}, k_{2}\right)$ over the region of support $k_{1}=0 \ldots N_{1}-1, k_{2}=$ $0 \ldots N_{2}-1$ in Fourier space[13]. Since in both physical and spectral spaces the discrete points are distributed at regular cartesian meshes, the minimal distances of these points will be used as an additional parameter of normalisation.

By using these notations, traditional DFT and inverse DFT (IDFT) write

$$
\begin{aligned}
& X^{F}\left(k_{1}, k_{2}\right)=\sum_{n_{1}=0}^{N_{1}-1} \sum_{n_{2}=0}^{N_{2}-1} x\left(n_{1}, n_{2}\right) W_{N_{1}}^{n_{1} k_{1}} W_{N_{2}}^{n_{2} k_{2}}, \\
& x\left(n_{1}, n_{2}\right)=\frac{1}{N_{1} N_{2}} \sum_{k_{1}=0}^{N_{1}-1} \sum_{k_{2}=0}^{N_{2}-1} X^{F}\left(k_{1}, k_{2}\right) W_{N_{1}}^{-n_{1} k_{1}} W_{N_{2}}^{-n_{2} k_{2}},
\end{aligned}
$$

with $W_{N}^{n k}=e^{-2 \pi i \frac{n k}{N}}$.

We then turn to the case of hexagonal Fourier transform. Supposing that there is a set of data sampled on a regular lattice of points in the physical space showed in Fig. 11(a) and 


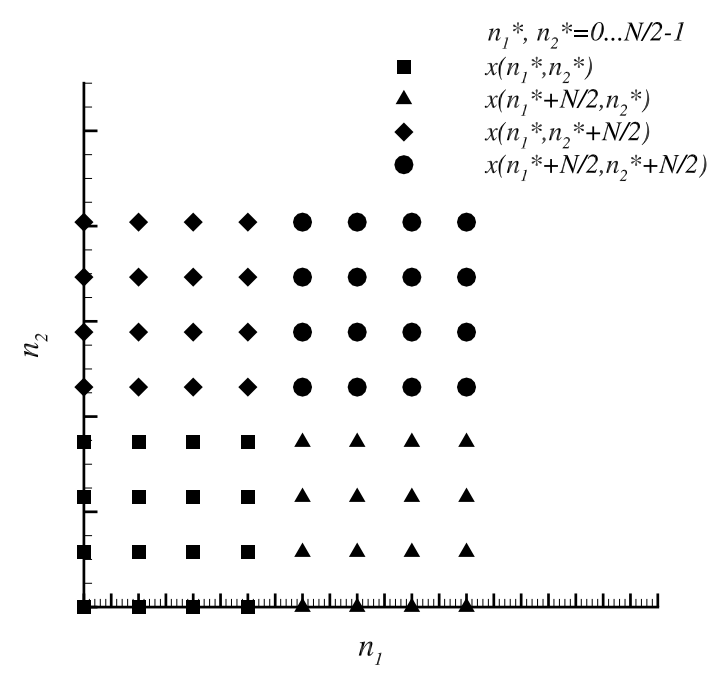

(a)

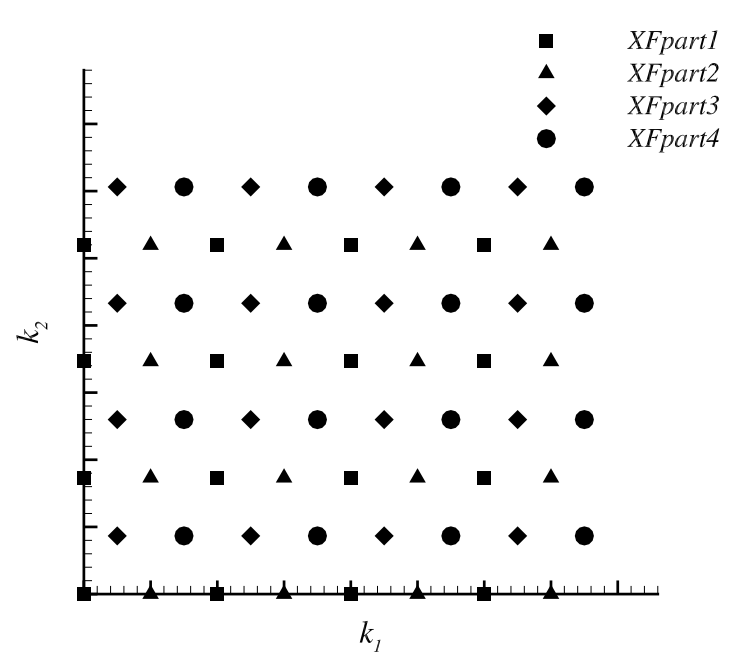

(b)

Figure 11. Sketch of the discrete points of two-dimensional hexagonal Fourier transform. (a) Sampling in physical space. (b) Discrete points in Fourier spectral space. In both spaces, the set of points are divided into four parts, which are treated separately in calculations.

its spectral value are located at the vertice of regular triangles in Fourier spectral space, as shown in Fig. 11(b). We classify the sample points in both physical and spectral spaces into four groups individually, denoted as the different symbols in Fig. 11. In fact, we can consider that in spectral space groups 3 and 4 are horizontally shifted from a cartesian mesh, and then extend the defining range of $X^{F}\left(k_{1}, k_{2}\right)$. We will use notation $X^{F}\left(k_{1}+\frac{1}{2}, k_{2}\right)$ to present the shifted points. For the aim of isotropy, in the present contribution we choose $N_{1}=N_{2}=N$. For the ease of FFT algorithm, the value of $N$ can be set as an positive integer power of 2 . Using the idea of decimation in frequency, we can rewrite formula (A1) as

$$
\begin{aligned}
X^{F}\left(k_{1}, k_{2}\right)=\sum_{n_{1}=0}^{\frac{N}{2}-1} \sum_{n_{2}=0}^{\frac{N}{2}-1}\left[x\left(n_{1}, n_{2}\right) W_{N}^{n_{1} k_{1}} W_{N}^{n_{2} k_{2}}\right. \\
+x\left(n_{1}+\frac{N}{2}, n_{2}\right)(-1)^{k_{1}} W_{N}^{n_{1} k_{1}} W_{N}^{n_{2} k_{2}} \\
+x\left(n_{1}, n_{2}+\frac{N}{2}\right)(-1)^{k_{2}} W_{N}^{n_{1} k_{1}} W_{N}^{n_{2} k_{2}} \\
\left.+x\left(n_{1}+\frac{N}{2}, n_{2}+\frac{N}{2}\right)(-1)^{k_{1}+k_{2}} W_{N}^{n_{1} k_{1}} W_{N}^{n_{2} k_{2}}\right] .
\end{aligned}
$$

in which the four terms of right hand side corresponds to the four groups of Fig. 11(a) 
respectively. Replacing the index of wavenumber $k_{1}$ and $k_{2}$ in Fourier space by introducing two new integer variables $p$ and $q$, where $p, q=0 \ldots \frac{N}{2}-1$, formula A2 is rewritten to four cases, corresponding to the four groups in Fig. 11(b):

$$
\begin{aligned}
& X^{F}(2 p, 2 q)=\sum_{n_{1}=0}^{\frac{N}{2}-1} \sum_{n_{2}=0}^{\frac{N}{2}-1}\left[x\left(n_{1}, n_{2}\right)+x\left(n_{1}+\frac{N}{2}, n_{2}\right)+x\left(n_{1}, n_{2}+\frac{N}{2}\right)\right. \\
& \left.+x\left(n_{1}+\frac{N}{2}, n_{2}+\frac{N}{2}\right)\right] W_{\frac{N}{2}}^{n_{1} p} W_{\frac{N}{2}}^{n_{2} q}, \\
& X^{F}(2 p+1,2 q)=\sum_{n_{1}=0}^{\frac{N}{2}-1} \sum_{n_{2}=0}^{\frac{N}{2}-1}\left[\left(x\left(n_{1}, n_{2}\right)-x\left(n_{1}+\frac{N}{2}, n_{2}\right)+x\left(n_{1}, n_{2}+\frac{N}{2}\right)\right.\right. \\
& \left.\left.-x\left(n_{1}+\frac{N}{2}, n_{2}+\frac{N}{2}\right)\right) W_{N}^{n_{1}}\right] W_{\frac{N}{2}}^{n_{1} p} W_{\frac{N}{2}}^{n_{2} q}, \\
& X^{F}\left(2 p+\frac{1}{2}, 2 q+1\right)=\sum_{n_{1}=0}^{\frac{N}{2}-1} \sum_{n_{2}=0}^{\frac{N}{2}-1}\left[\left(x\left(n_{1}, n_{2}\right)+x\left(n_{1}+\frac{N}{2}, n_{2}\right) W_{N}^{\frac{N}{4}}-x\left(n_{1}, n_{2}+\frac{N}{2}\right)\right.\right. \\
& \left.\left.-x\left(n_{1}+\frac{N}{2}, n_{2}+\frac{N}{2}\right) W_{N}^{\frac{N}{4}}\right) W_{N}^{n_{2}} W_{N}^{\frac{n_{1}}{2}}\right] W_{\frac{N}{2}}^{n_{1} p} W_{\frac{N}{2}}^{n_{2} q}, \\
& X^{F}\left(2 p+\frac{3}{2}, 2 q+1\right)=\sum_{n_{1}=0}^{\frac{N}{2}-1} \sum_{n_{2}=0}^{\frac{N}{2}-1}\left[\left(x\left(n_{1}, n_{2}\right)-x\left(n_{1}+\frac{N}{2}, n_{2}\right) W_{N}^{\frac{N}{4}}-x\left(n_{1}, n_{2}+\frac{N}{2}\right)\right.\right. \\
& \left.\left.+x\left(n_{1}+\frac{N}{2}, n_{2}+\frac{N}{2}\right) W_{N}^{\frac{N}{4}}\right) W_{N}^{n_{2}} W_{N}^{\frac{3 n_{1}}{2}}\right] W_{\frac{N}{2}}^{n_{1} p} W_{\frac{N}{2}}^{n_{2} q} .
\end{aligned}
$$

For the last two equations where we calculate the spectral value of the positions that are not the vertice of rectangular, we performed a cyclic translation which is a property of Fourier transformation. Note that by reconstructing these four new sequences of data with $\frac{N}{2} * \frac{N}{2}$ points in physical field, we can use the FFT algorithm on each of them to calculate different parts of the discrete Fourier transform in the form of hexagonal. For example, in order to calculate the first part in Fourier space, the new sequence $x_{1}$ to be used is defined as $x_{1}\left(n_{1}, n_{2}\right)=x\left(n_{1}, n_{2}\right)+x\left(n_{1}+\frac{N}{2}, n_{2}\right)+x\left(n_{1}, n_{2}+\frac{N}{2}\right)+x\left(n_{1}+\frac{N}{2}, n_{2}+\frac{N}{2}\right)$ where the ranges of two integers are $n_{1}, n_{2}=0 \ldots \frac{N}{2}-1$, thus we have

$$
X^{F}(2 p, 2 q)=X_{1}^{F}(p, q), \quad X_{1}^{F}=\operatorname{FFT}\left(x_{1}\right)
$$

The other parts of the discrete Fourier transform $X_{2}^{F}, X_{3}^{F}, X_{4}^{F}$ can be calculated similarly. As a result, we can obtain the hexagonal spectrum by performing four times the FFT algorithms to the arranged sequences of data in physical field.

The inverse operation uses a similar idea. For a given hexagonal discrete spectrum $X^{F}$ in Fourier space, we also have to abstract four parts. We do IFFT and obtain four groups 
of points. Then we reconstruct the physical sampling for the lattice of points by resolving a system of equations. In details, for example, we have for the first sequence obtained by the inverse fast Fourier transform with $X_{1}^{F}(p, q)=X^{F}(2 p, 2 q)$ for $p, q=0 \ldots \frac{N}{2}-1$ and for $n_{1}, n_{2}=0 \ldots \frac{N}{2}-1$

$$
\operatorname{IFFT}\left(X_{1}^{F}\right)=x_{1}
$$

where

$$
x_{1}\left(n_{1}, n_{2}\right)=x\left(n_{1}, n_{2}\right)+x\left(n_{1}+\frac{N}{2}, n_{2}\right)+x\left(n_{1}, n_{2}+\frac{N}{2}\right)+x\left(n_{1}+\frac{N}{2}, n_{2}+\frac{N}{2}\right) .
$$

After having the four sequences $x_{1}, x_{2}, x_{3}, x_{4}$, we can restitute the sequence of values $x$ at the initial positions which means at the vertices of rectangular. For $n_{1}, n_{2}=0 \ldots \frac{N}{2}-1$

$$
\begin{gathered}
x\left(n_{1}, n_{2}\right)=\frac{1}{4}\left[x_{1}\left(n_{1}, n_{2}\right)+W_{N}^{-n_{1}} x_{2}\left(n_{1}, n_{2}\right)\right. \\
\left.+W_{N}^{-\frac{n_{1}}{2}} W_{N}^{-n_{2}} x_{3}\left(n_{1}, n_{2}\right)+W_{N}^{-\frac{n_{3}}{2}} W_{N}^{-n_{2}} x_{4}\left(n_{1}, n_{2}\right)\right] \\
x\left(n_{1}, n_{2}+\frac{N}{2}\right)=\frac{1}{4}\left[x_{1}\left(n_{1}, n_{2}\right)+W_{N}^{-n_{1}} x_{2}\left(n_{1}, n_{2}\right)\right. \\
\left.\quad-W_{N}^{-\frac{n_{1}}{2}} W_{N}^{-n_{2}} x_{3}\left(n_{1}, n_{2}\right)-W_{N}^{-\frac{n_{3}}{2}} W_{N}^{-n_{2}} x_{4}\left(n_{1}, n_{2}\right)\right] \\
x\left(n_{1}+\frac{N}{2}, n_{2}\right)=\frac{1}{4}\left[x_{1}\left(n_{1}, n_{2}\right)-W_{N}^{-n_{1}} x_{2}\left(n_{1}, n_{2}\right)\right. \\
\left.\quad+W_{N}^{-\frac{n_{1}}{2}} W_{N}^{-n_{2}} W_{N}^{-\frac{N}{4}} x_{3}\left(n_{1}, n_{2}\right)-W_{N}^{-\frac{n_{3}}{2}} W_{N}^{-n_{2}} W_{N}^{-\frac{N}{4}} x_{4}\left(n_{1}, n_{2}\right)\right] \\
x\left(n_{1}+\frac{N}{2}, n_{2}+\frac{N}{2}\right)=\frac{1}{4}\left[x_{1}\left(n_{1}, n_{2}\right)-W_{N}^{-n_{1}} x_{2}\left(n_{1}, n_{2}\right)\right. \\
\left.\quad-W_{N}^{-\frac{n_{1}}{2}} W_{N}^{-n_{2}} W_{N}^{-\frac{N}{4}} x_{3}\left(n_{1}, n_{2}\right)+W_{N}^{-\frac{n_{3}}{2}} W_{N}^{-n_{2}} W_{N}^{-\frac{N}{4}} x_{4}\left(n_{1}, n_{2}\right)\right]
\end{gathered}
$$

Then we obtain the values of the physical field for a sequence of data on a lattice of points.

Using four times FFT/IFFT algorithm can reduce efficiently the cost of computation compared with constructing the circulations in the algorithm. We usually use only the multiple of the step length in both two space in the formulas because the period value can be eliminated by choosing properly the position of spectrum to be calculated.

For a finite field of discrete spectrum, the inverse operation for the purpose of reconstructing a whole field or a larger physical field has the coincidence with the inverse operation of Fourier transformation continuous in which we also have a summation to be calculated The only difference is the occurrence of a constant multiplier associated with the number of sample points chosen during the calculation of the discrete spectrum. 
In brief, for the hexagonal Fourier transform, we can take advantage of the modified FFT and IFFT algorithms, including their parallelized versions, to save the programming and computational costs in the pseudo-spectral methods of turbulence simulations.

[1] R.S. Rogallo. Numerical experiments in homogeneous turbulence. NASA TM 81315, 1981.

[2] B. Thornber. Impact of domain size and statistical errors in simulations of homogeneous decaying turbulence and the Richtmyer-Meshkov instability. Physics of Fluids, 28:045106, 2016.

[3] L Fang. Background scalar-level anisotropy caused by low-wave-number truncation in turbulent flows. Physical Review E, 95(3):033102, 2017.

[4] T. Watanabe and T. Gotoh. Statistics of a passive scalar in homogeneous turbulence. New Journal of Physics, 6:40, 2004.

[5] Y. Li, E. Perlman, M. Wan, Y. Yang, R. Burns, C. Meneveau, S. Chen, A. Szalay, and G. Eyink. A public turbulence database cluster and applications to study lagrangian evolution of velocity increments in turbulence. Journal of Turbulence, 9(31), 2008.

[6] R. Samtaney, D.I. Pullin, and B. Kosovic. Direct numerical simulation of decaying compressible turbulence and shocklet statistics. Physics of Fluids, 13(5):1415-1430, 2001.

[7] F. Liu, L.P. Lu, W.J.T. Bos, and L. Fang. Assessing the nonequilibrium of decaying turbulence with reversed initial fields. Physical Review Fluids, 4:084603, 2019.

[8] Z.C. Qin, L. Fang, and J. Fang. How isotropic are turbulent flows generated by using periodic conditions in a cube? Physics Letters A, 380(13):1310-1317, 2016.

[9] F. Waleffe. The nature of triad interactions in homogeneous turbulence. Physics of Fluids A, 4(2):350-363, 1992.

[10] Ö.D. Gürcan. Nested polyhedra model of turbulence. Physical Review E, 95(6):063102, 2017.

[11] Ö.D. Gürcan. Nested polyhedra model of isotropic magnetohydrodynamic turbulence. Physical Review E, 97(6):063111, 2018.

[12] P.K. Murphy and N.C. Gallagher. Hexagonal sampling techniques applied to Fourier and Fresnel digital holograms. Optical Society of America, 72(7):929-937, 1982.

[13] J.C. Ehrhardt. Hexagonal fast fourier transform with rectangular output. IEEE Transactions on Signal Processing, 41(3):1469-1472, 1993. 
[14] A.P. Fitz and R.J. Green. Fingerprint classification using a hexagonal fast fourier transform. Pattern Recognition, 29:1587-1597, October 1996.

[15] A.M. Grigoryan. Efficient algorithms for computing the 2-D hexagonal Fourier transforms. IEEE Transactions on Signal Processing, 50:1438-1448, June 2002.

[16] J.B. Birdsong and N.I. Rummelt. The hexagonal fast fourier transform. In 2016 IEEE International Conference on Image Processing (ICIP), pages 1809-1812, Phoenix, AZ, USA, September 2016. IEEE.

[17] R. Stepanov and F. Plunian. Systematic bias in the calculation of spectral density from a three-dimensional spatial grid. Physical Review E, 90:053309, 2014.

[18] W.J.T. Bos, L. Chevillard, J. Scott, and R. Rubinstein. Reynolds number effects on the velocity increment skewness in isotropic turbulence. Physics of Fluids, 24:015108, 2012.

[19] M. Lesieur. Turbulence in Fluids. Kluwer Academic, Dordrecht, 1997.

[20] S. A. Orszag. Lectures on the statistical theory of turbulence. 1974.

[21] R.H. Kraichnan. The structure of isotropic turbulence at very high Reynolds numbers. Journal of Fluid Mechanics, 5:497-543, 1959.

[22] L. Fang, W.J.T. Bos, and G.D. Jin. Short-time evolution of lagrangian velocity gradient correlations in isotropic turbulence. Physics of Fluids, 27:125102, 2015.

[23] K. Ohkitani and M. Yamada. Temporal intermittency in the energy cascade process and local Lyapunov analysis in fully-developed model turbulence. Progress of Theoretical Physics, 81:329, 1989 .

[24] V.S. L'vov, E. Podivilov, A. Pomyalov, I. Procaccia, and D. Vandembroucq. Improved shell model of turbulence. Physical Review E, 58(2):1811-1822, 1998.

[25] L. Biferale. Shell models of energy cascade in turbulence. Annual Review of Fluid Mechanics, 35(1):441-468, 2003.

[26] H.K. Moffatt. The degree of knottedness of tangled vortex lines. Journal of Fluid Mechanics, 35:117-129, 1969 .

[27] J.Z. Zhu. Vorticity and helicity decompositions and dynamics with real Schur form of the velocity gradient. Physical of Fluids, 30:031703, 2018. 